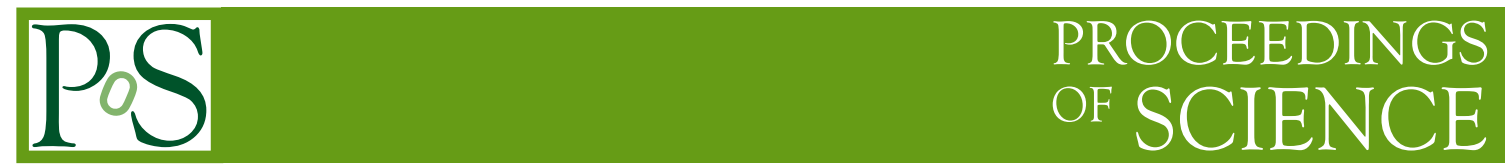

\title{
Precision QCD measurements at the LHC
}

\section{Piergiulio Lenzi ${ }^{* \dagger}$}

Istituto Nazionale di Fisica Nucleare (Sez. Firenze)

E-mail: piergiulio.lenzi@cern.ch

After the successful end of the LHC Run 1, culminating in the discovery of a new boson, we review the status of the QCD measurements performed by the two multi purpose experiments, ATLAS and CMS, putting into evidence the precision of those measurements and their relevance for the advancement in the understanding of proton-proton collisions phenomenology.

XXI International Workshop on Deep-Inelastic Scattering and Related Subject -DIS2013, 22-26 April 2013

Marseilles, France

\footnotetext{
* Speaker.

†n behalf of the ATLAS and CMS collaborations.
} 


\section{Introduction}

A detailed understanding of QCD phenomenology is a prerequisite for any search for Standard Model and New Physics at the LHC. On the one hand studying the abundant QCD processes at the LHC gives confidence in the detector performance and reconstruction techniques, on the other hand it gives relevant information to improve our understanding of QCD phenomenology. QCD processes were studied in great detail with a large set of observables, ranging from very inclusive to very exclusive quantities. The study of different quantities gave access to different ingredients of perturbative QCD phenomenology. While rather inclusive observables provide important constraints on parton distribution functions, probing differentially multi-jet final states puts into evidence the strengths and weaknesses of different approaches used to describe multi-particles final states. In this note we will concentrate on the review of the recent measurements that are particularly relevant in the comparison with the predictions of different parton shower, fixed order and hybrid fixed order plus parton shower calculations.

This note is organized as follows. In Sec. 2 the status of inclusive jets measurements is reviewed and compared to state of the art theoretical tools. In Sec. 3, moving one step forward in the exclusiveness of the final state, the status of di-jet measurements is reviewed. Finally in Sec. 4 the status of vector boson plus jets is reviewed, starting from very inclusive observables and gradually moving the focus to more exclusive final states.

\section{Inclusive jets}

The ATLAS [1] collaboration, using $37 \mathrm{pb}^{-1}$ of luminosity from the 2010 data taking, measured the inclusive jet spectra for jets with transverse momentum $\left(\mathrm{p}_{\mathrm{T}}\right)$ larger than $20 \mathrm{GeV}$, in six bins of jet rapidity (up to 4.4 units) [2]. The measurement showed good agreement with next-toleading order (NLO) perturbative QCD (implemented in the NLOJet++ [3] parton level generator, using modern parton density functions (PDFs) [4]), with a slight tendency of the predictions to overestimate the cross section especially at central rapidities. This measurement also showed good agreement with the predictions of NLO plus parton shower (PS) implemented in the POWHEG generator [5]. The CMS [6] collaboration made a similar measurement using the full statistics collected in $2011,5 \mathrm{fb}^{-1}$ [7], for jets with rapidity up to 2.5 units. This measurement also shows good agreement with the predictions of perturbative QCD and puts into evidence the impact that this kind of measurement can have on PDF fits, the experimental errors being of the same order of the theoretical uncertainty. The ATLAS collaboration has also released a comparison between inclusive jet spectra at $7 \mathrm{TeV}$ and at $2.76 \mathrm{TeV}$ center of mass energy, taking advantage of a short LHC run at $2.76 \mathrm{TeV}$ [8]. Taking the ratio of spectra at the two energies allows partial or total cancellation of several experimental uncertainties, yielding a very precise result, that can be useful especially to constrain PDF fits. A subset of the plots shown in the paper is reported in Fig. 1, where the $2.76 \mathrm{TeV}$ over $7 \mathrm{TeV}$ cross section ratio is divided by the prediction of parton level NLO QCD and NLO QCD plus PS (POWHEG) showing a certain amount of disagreement especially at central rapidities. 

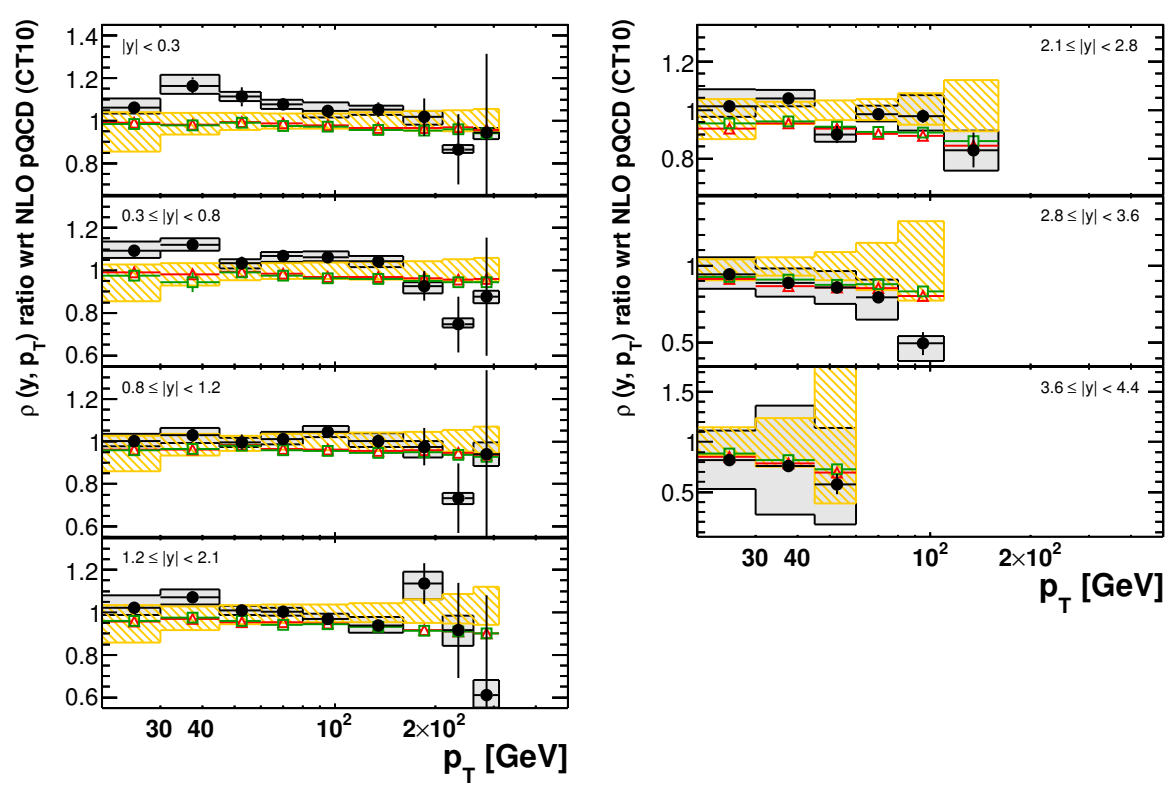

ATLAS

$\int L \mathrm{dt}=0.20 \mathrm{pb}^{-1}$

$\rho=\sigma_{\text {jet }}^{2.76 \mathrm{TeV}} / \sigma_{\text {jet }}^{\text {7TeV }}$

anti- $k_{t} R=0.4$

Data with

statistical

uncertainty

Systematic

uncertainties NLO PQCD $\otimes$ non-pert. corr. $\left(\mathrm{CT} 10, \mu=p_{\mathrm{T}}^{\max }\right)$ POWHEG $\otimes$ PYTHIA

$\triangle \quad$ tune AUET2B $\left(\mathrm{CT} 10, \mu=p_{T}^{\text {Borm }}\right)$ POWHEG $\otimes$ PYTHIA

—- tune Perugia 2011 $\left(\mathrm{CT} 10, \mu=p_{\mathrm{T}}^{\text {Borm }}\right)$

Figure 1: $2.76 \mathrm{TeV}$ over $7 \mathrm{TeV}$ cross section ratio in data divided by the NLO perturbative QCD prediction. The comparison is shown in seven bins of jet rapidity. The comparison with NLO QCD plus PS implemented in POWHEG is also shown. The different POWHEG curves correspond to different tunes of the PS part.

\section{Di-jets and multi-jets}

Several results on final states with at least two jets have been released recently. One of the most relevant ones is the measurement of the value of the strong coupling constant $\alpha_{\mathrm{s}}$.

ATLAS and CMS used similar observables to measure this quantity. ATLAS in [9] used the ratio $\left(\mathrm{N}_{3 / 2}\right)$ of the inclusive jet $\mathrm{p}_{\mathrm{T}}$ spectrum obtained by requiring at least three jets over the one obtained requiring at least two jets. Jets selected for the ATLAS analysis had $\mathrm{p}_{\mathrm{T}}$ higher than $40 \mathrm{GeV}$, with the exception of the leading jet which had a minimum $\mathrm{p}_{\mathrm{T}}$ requirement of $60 \mathrm{GeV}$. CMS in [10] used the ratio $\left(\mathrm{R}_{32}\right)$ of the number of events with at least three jets over the number of events with at least two jets as a function of the average $\mathrm{p}_{\mathrm{T}}$ of the two leading jets. Jets selected for the CMS analysis had a minimum $\mathrm{p}_{\mathrm{T}}$ requirement of $150 \mathrm{GeV}$. The measurement from ATLAS was performed using the $201037 \mathrm{pb}^{-1}$ dataset, while the CMS measurement was performed using the $20115 \mathrm{fb}^{-1}$ dataset. The sensitivity of the observables studied by the two experiments to the value of $\alpha_{\mathrm{s}}$ is shown in Fig. 2 for ATLAS (a) and CMS (b). The value of $\alpha_{\mathrm{s}}$ was obtained in both analyses by fitting Monte Carlo templates, obtained with different values of $\alpha_{\mathrm{s}}$, to the measured spectra. The ATLAS analysis yielded a value of $\alpha_{\mathrm{s}}=0.111 \pm 0.006(\exp )_{-0.003}^{+0.016}$ (theory), while the CMS result is $\alpha_{\mathrm{s}}=0.1148 \pm 0.0014$ (exp.) \pm 0.0018 (PDF) ${ }_{-0.0000}^{+0.0050}$ (scale). Most part of the difference in the theoretical error comes from the different scale uncertainty, which is much smaller in CMS than in ATLAS. The source of this difference is most likely related to the higher $\mathrm{p}_{\mathrm{T}}$ threshold in the CMS analysis, which makes it less sensitive to scale variations. 


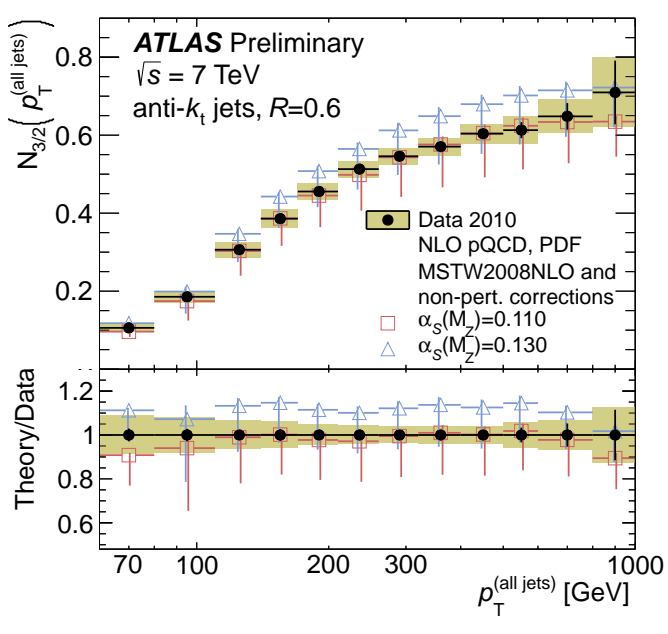

(a)

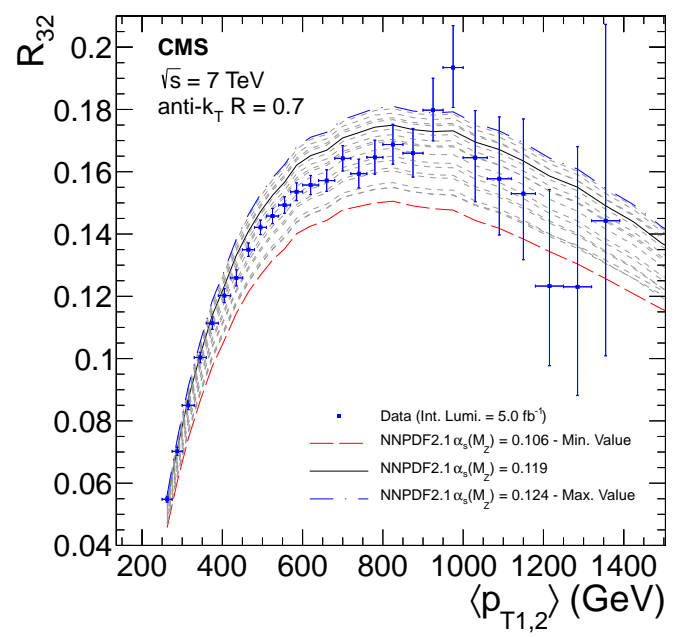

(b)

Figure 2: The sensitivity of ATLAS $N_{3 / 2}$ observable (a) and CMS $\mathrm{R}_{32}$ observable to the value of $\alpha_{\mathrm{s}}$ is shown.

\section{Vector boson plus jets}

In this section we review the status of recent measurements of photon plus jets and W/Z plus jets.

Photon plus jets final states have been studied recently by both ATLAS [11] and CMS [12]. Both experiments observe nice agreement between data and predictions of parton level perturbative QCD at NLO (JETPHOX [13]) and leading order (LO) plus PS generator (Sherpa [14]), as observed for example in Fig. 3, where the CMS collaboration shows the $\mathrm{p}_{\mathrm{T}}$ spectrum of the photon in four

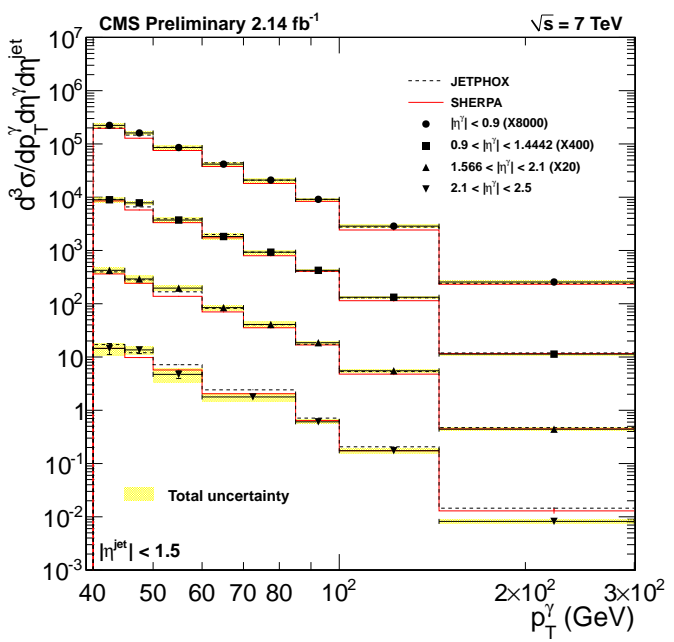

(a)

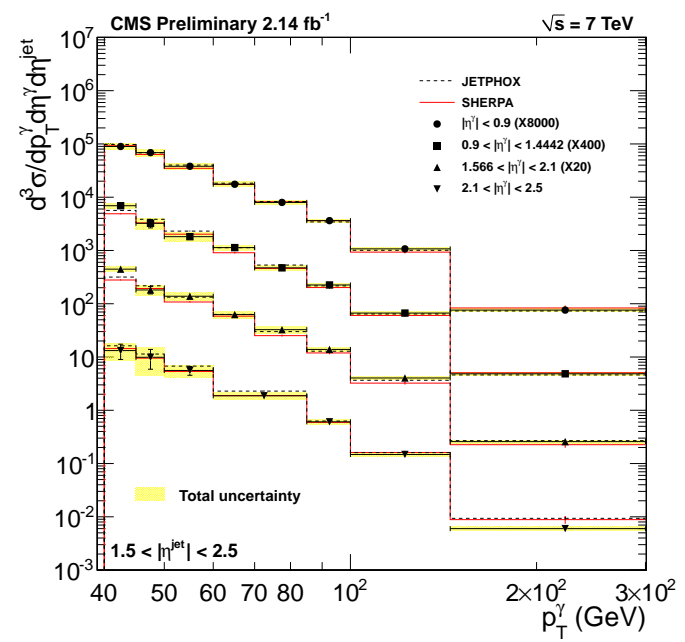

(b)

Figure 3: Photon $\mathrm{p}_{\mathrm{T}}$ spectrum in four bins of photon rapidity and two bins of jet rapidity $\left(\eta^{\text {jet }}\right)$. (a) corresponds to $\left|\eta^{\text {jet }}\right|<1.5$, (b) corresponds to $1.5<\left|\eta^{\text {jet }}\right|<2.5$ 


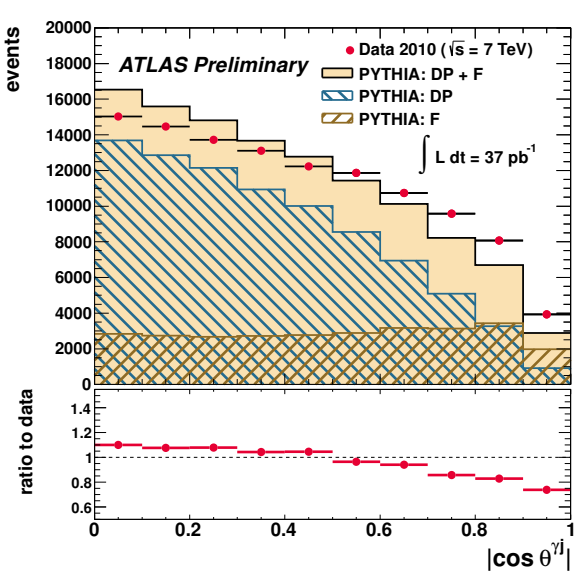

(a)

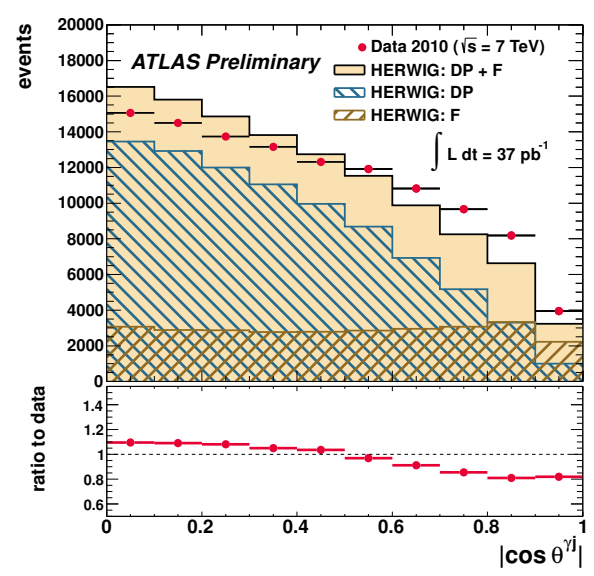

(b)

Figure 4: Comparison of data and PS predictions for $\theta^{\gamma \mathrm{j}}$, showing explicitly the fragmentation (F) and direct (DP) components in the prediction. Data are compared to PYTHIA in (a) and to HERWIG in (b).

bins of photon rapidity and two bins of leading jet rapidity. The ATLAS collaboration has put into evidence the different contributions of direct and fragmentation photons. This is particularly visible in the scattering angle $\left(\theta^{\gamma \mathrm{j}}\right)$ of the photon with respect to the beam line in the photon-leading jet rest frame, as shown in Fig.4, where data are compared to the predictions of PYTHIA [15] and HERWIG [16], separating explicitly the fragmentation (F) and direct (DP) components of the prediction. The comparison shows that pure PS programs are unable to get the right fraction of the two components. It is also shown in [11] that reasonable agreement can be achieved by rescaling the two components to get the best fit of the data. Other photon plus jet related observables, mainly focusing on angular correlations between the photon and the jet, have been reported recently in [17], where the ATLAS collaboration studied a fragmentation enhanced region in which the sign of the jet and photon rapidities is the same, and in [18], where the CMS collaboration studied the rapidity difference between the photon and the jet (and similarly between a $\mathrm{Z}$ boson and a jet), which seems rather sensitive to the details of how the matrix element and the PS are put together in a LO plus PS calculation.

Final states with a $\mathrm{W}$ or a $\mathrm{Z}$ boson plus additional QCD radiation have been studied extensively, and both ATLAS and CMS have produced new results recently. A variety of observables have been used to characterize these final states, only a few will be discussed here in some detail. One of the most inclusive observables one can think of to characterize the QCD radiation produced in association with a $\mathrm{Z}$ boson is the $\mathrm{Z}$ boson $\mathrm{p}_{\mathrm{T}}$. Recently, this has been measured directly by CMS for $\mathrm{Z}$ bosons decaying to electrons or muons at $8 \mathrm{TeV}$ center-of-mass energy [19]. An observable, called $\phi_{\eta}^{*}$, that is strongly correlated with the $\mathrm{p}_{\mathrm{T}}$, has been measured by ATLAS [20]. The $\phi_{\eta}^{*}$ is essentially the scattering angle between the two leptons from $\mathrm{Z}$ decay in the $\mathrm{Z}$ rest frame. Compared to the $\mathrm{Z}$ boson $\mathrm{p}_{\mathrm{T}}, \phi_{\eta}^{*}$ can be measured more precisely because it is defined only by means of angular variables. The conclusion that can be drown from these studies is that $\mathrm{Z} \mathrm{p}_{\mathrm{T}}$ related observables are extremely sensitive to the details of the description of QCD radiation, especially the soft 


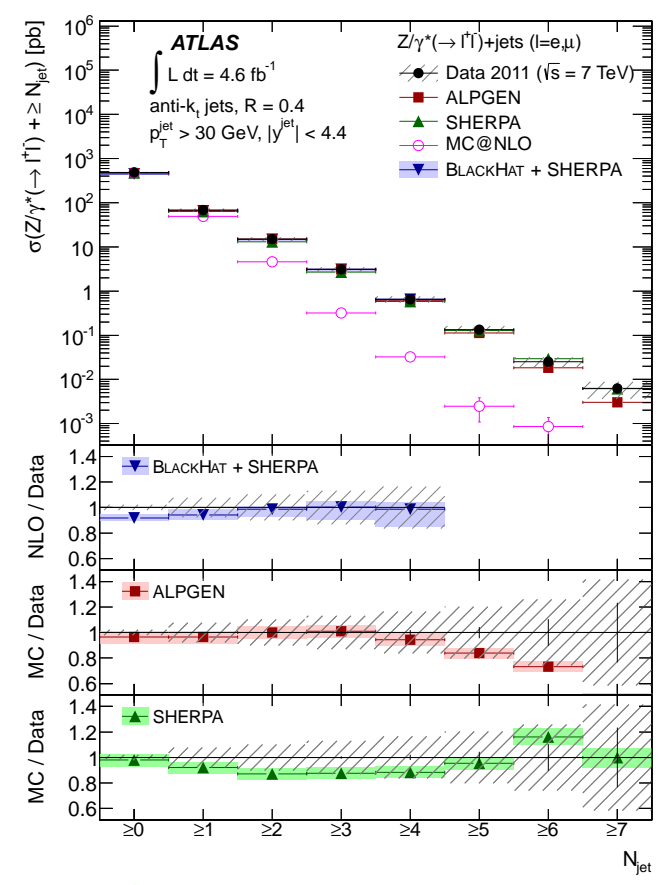

(a)

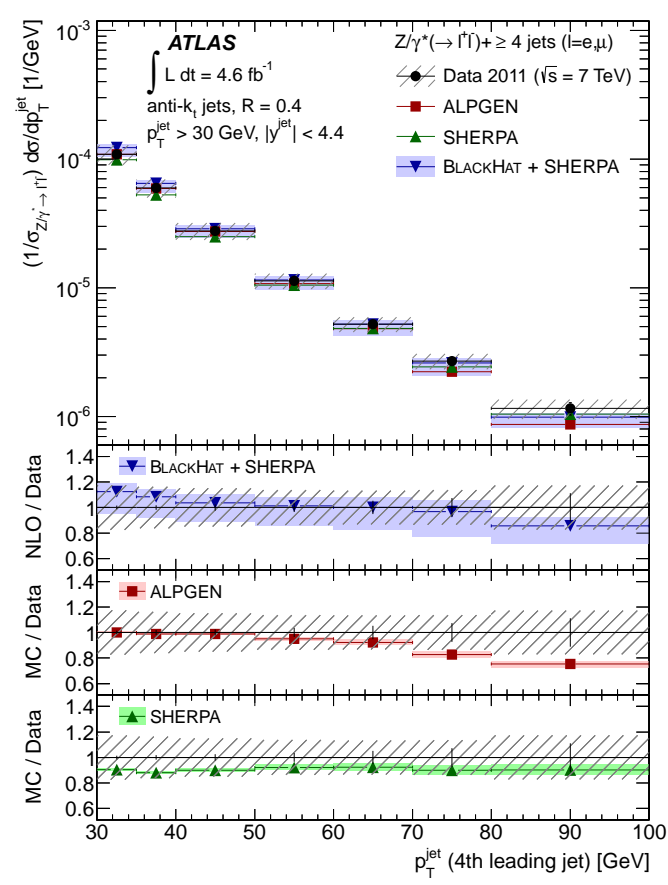

(b)

Figure 5: Jet multiplicity spectrum (a) and fourth jet $\mathrm{p}_{\mathrm{T}}$ spectrum (b) in Z+jets.

and non perturbative part of it. None of the models that have been tried by the two experiments, ranging from pure PS programs with different underlying event models (PYTHIA, HERWIG) to analytically resummed calculation (RESBOS [21]) is able to describe the whole spectrum.

ATLAS and CMS looked also into more differential observables in $\mathrm{W}$ and $\mathrm{Z}$ plus jets. Both experiments published several differential measurements using using the $201037 \mathrm{pb}^{-1}$ sample [22, 23]. Recently the ATLAS collaboration has measured several $Z+j e t s$ related observables using the full $20115 \mathrm{fb}^{-1}$ sample [24]. As an example the jet multiplicity spectrum and the $\mathrm{p}_{\mathrm{T}}$ of the fourth jet in Z+jets is shown in Fig. 5 (a) and (b) respectively. Data are compared to the prediction of several calculations. MC@NLO [25] is an NLO calculation of inclusive Z production matched to PS; it is expected to get the first jet at LO precision and to underestimate the rate for more than one jet. Alpgen [26] and Sherpa are LO plus PS calculation that are expected to describe with LO accuracy multi jet final states; they perform very well in this comparison. Finally BlackHat [27] is a NLO parton level calculation for up to five jets; which also shows very nice agreement with the data.

Another way the two collaboration have pursued to characterize W/Z plus jets is through event shapes. ATLAS has recently measured $k_{T}$ splitting scales in $\mathrm{W}+$ jets [28]. This observable is extremely powerful, as it provides a detailed description of the QCD radiation accompanying the vector boson, being sensitive both to the jet structure and to the hard emission. Several models have been compared to the data. LO plus PS generators show very nice description of multi jet final states, while the region where the observable is most sensitive to collinear radiation is best described by HERWIG. CMS studied a different event shape observable, the central transverse 


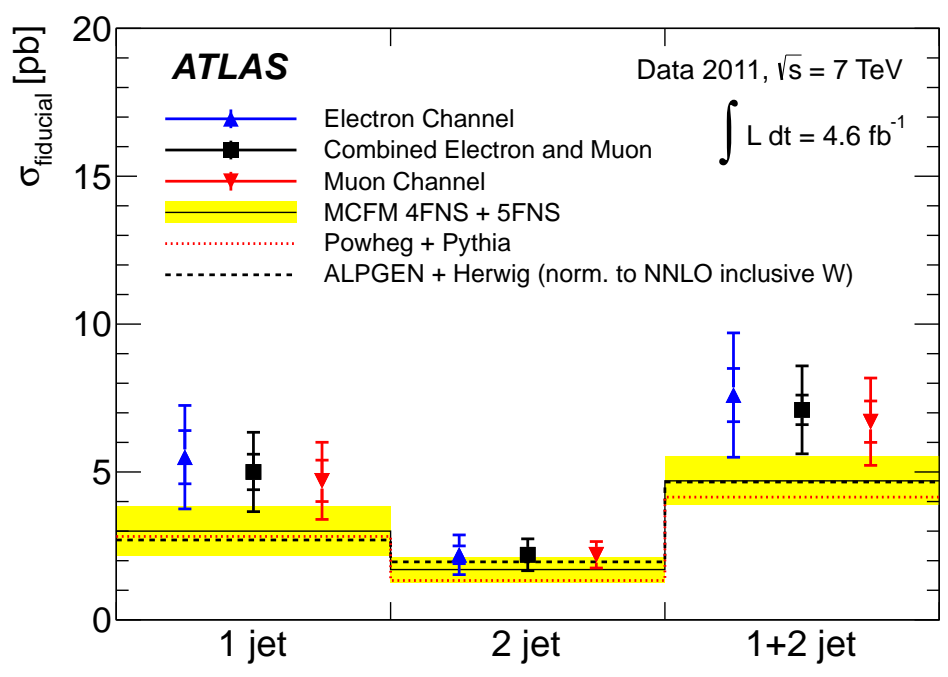

Figure 6: Cross section for $\mathrm{W}$ plus exactly one b-jet, exactly two b-jets and the combination of the two, compared to theoretical predictions.

thrust [29], which is defined similarly to the e+e- collider thrust, but using only transverse components and only using jet momenta and the $\mathrm{Z}$ boson momentum as input. This observable also bridges the regimes of collinear radiation and multi-jet final states, thus giving a very comprehensive picture of QCD radiation in this kind of events. Nice agreement is observed with the prediction of $\mathrm{Z}+1$ jet at NLO matched with PS, as implemented in POWHEG [30].

Several recent measurement have been carried out to study the heavy flavor composition of the jets produced in association with a $\mathrm{W}$ or a $\mathrm{Z}$ boson. ATLAS has characterized $\mathrm{W}$ plus at least one b-jet production in [31], using the full 2011 dataset. The comparison of the measured cross section with predictions is shown in Fig. 6, for events with exactly one b-jet in a fiducial region, exactly two b-jets in the same fiducial region, and the combination of the two. All predictions tend to undershoot slightly the data. On the other hand, a characterization of W plus two b-jets recently released by CMS [32] shows good agreement with predictions. Studies of associated production of $\mathrm{a} Z \mathrm{Z}$ boson and $\mathrm{b}$-jets have also been carried out. ATLAS measured the production cross section for $\mathrm{Z}$ plus at least one b-jet in [33]. CMS studied the production of a $\mathrm{Z}$ boson and two b-jets in [34]. Good agreement is found with the prediction of NLO QCD and with the predictions of LO plus PS generators once the cross section for inclusive $\mathrm{Z}$ production is scaled with a global LO to NLO k-factor. As an example, Fig. 7 shows the CMS measurement of the $b \bar{b}$ system $\mathrm{p}_{\mathrm{T}}$, compared with the predictions of LO plus PS obtained with Madgraph [35] showered with PYTHIA.

\section{Conclusion}

Thanks to an extremely successful LHC Run 1 ATLAS and CMS have collected a large amount of data that, together with detailed understanding of the two apparatuses, has allowed the two collaborations to carry on precision QCD measurements for a variety of observables, ranging from very inclusive to very exclusive final states, from low to high $\mathrm{p}_{\mathrm{T}}$ observables. These measurements 


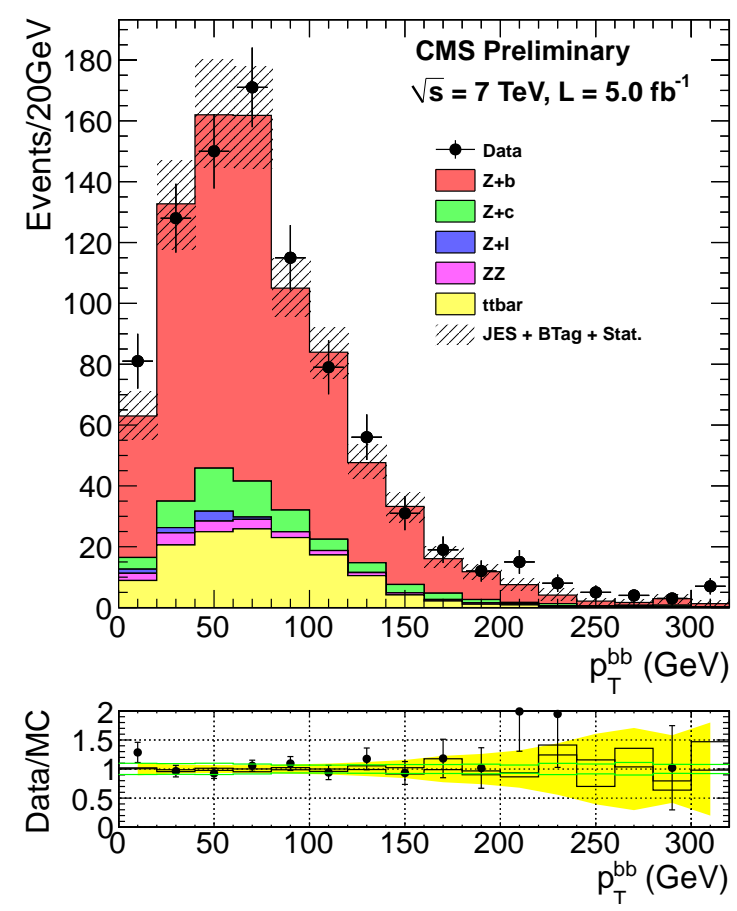

Figure 7: $\mathrm{p}_{\mathrm{T}}$ of the $b \bar{b}$ system produced in association with a $\mathrm{Z}$ boson.

have improved our understanding of QCD in several ways. They have allowed detailed comparison with the predictions of modern calculations and event generators, with experimental errors that in several cases are of the same order or smaller of the corresponding theory errors.

\section{References}

[1] ATLAS Collaboration, G. Aad et al., "The ATLAS Experiment at the CERN Large Hadron Collider", JINST 3 (2008) S08003.

[2] ATLAS Collaboration, G. Aad et al., "Measurement of inclusive jet and dijet production in $p p$ collisions at $\sqrt{s}=7 \mathrm{TeV}$ using the ATLAS detector", Phys.Rev. D86 (2012) 014022, arXiv:1112.6297 [hep-ex].

[3] Z. Nagy, "Next-to-leading order calculation of three jet observables in hadron hadron collision", Phys.Rev. D68 (2003) 094002, arXiv: hep-ph/ 0307268 [hep-ph] .

[4] H.-L. Lai, M. Guzzi, J. Huston, Z. Li, P. M. Nadolsky, et al., "New parton distributions for collider physics", Phys.Rev. D82 (2010) 074024, arXiv:1007.2241 [hep-ph] .

[5] S. Alioli, K. Hamilton, P. Nason, C. Oleari, and E. Re, "Jet pair production in POWHEG", JHEP 1104 (2011) 081, arXiv:1012.3380 [hep-ph].

[6] CMS Collaboration, S. Chatrchyan et al., "The CMS experiment at the CERN LHC”, JINST 3 (2008) S08004.

[7] CMS Collaboration, S. Chatrchyan et al., "Measurements of differential jet cross sections in proton-proton collisions at $\sqrt{s}=7 \mathrm{TeV}$ with the CMS detector", Phys.Rev. D87 (2013) 112002 , arXiv:1212.6660 [hep-ex]. 
[8] ATLAS Collaboration, G. Aad et al., "Measurement of the inclusive jet cross section in pp collisions at $\sqrt{s}=2.76 \mathrm{TeV}$ and comparison to the inclusive jet cross section at $\sqrt{s}=7 \mathrm{TeV}$ using the ATLAS detector", arXiv:1304.4739 [hep-ex].

[9] ATLAS Collaboration, "Measurement of multi-jet cross-section ratios and determination of the strong coupling constant in proton-proton collisions at sqrt(s)=7 TeV with the ATLAS detector.", Tech. Rep. ATLAS-CONF-2013-041, CERN, Geneva, Apr, 2013.

[10] CMS Collaboration, S. Chatrchyan et al., "Measurement of the ratio of the inclusive 3-jet cross section to the inclusive 2-jet cross section in pp collisions at $\sqrt{s}=7 \mathrm{TeV}$ and first determination of the strong coupling constant in the TeV range", arXiv:1304.7498 [hep-ex].

[11] ATLAS Collaboration, "Dynamics of isolated-photon and jet production in $p p$ collisions at $\sqrt{s}=7$ TeV with the ATLAS detector”, Tech. Rep. ATLAS-CONF-2013-023, CERN, Geneva, Mar, 2013.

[12] CMS Collaboration , "Measurement of triple-differential cross section of gamma+jet production", Tech. Rep. CMS-PAS-QCD-11-005, CERN, Geneva, 2013.

[13] S. Catani, M. Fontannaz, J. Guillet, and E. Pilon, "Cross-section of isolated prompt photons in hadron hadron collisions", JHEP 0205 (2002) 028, arXiv: hep-ph/0204023 [hep-ph] .

[14] T. Gleisberg, S. Hoeche, F. Krauss, M. Schonherr, S. Schumann, et al., "Event generation with SHERPA 1.1”, JHEP 0902 (2009) 007, arXiv:0811.4622 [hep-ph] .

[15] T. Sjostrand, S. Mrenna, and P. Z. Skands, "PYTHIA 6.4 Physics and Manual”, JHEP 0605 (2006) 026, arXiv:hep-ph/0603175 [hep-ph].

[16] G. Corcella, I. Knowles, G. Marchesini, S. Moretti, K. Odagiri, et al., "HERWIG 6: An Event generator for hadron emission reactions with interfering gluons (including supersymmetric processes)", JHEP 0101 (2001) 010, arXiv: hep-ph/0011363 [hep-ph] .

[17] ATLAS Collaboration, G. Aad et al., "Measurement of the production cross section of an isolated photon associated with jets in proton-proton collisions at $\sqrt{s}=7 \mathrm{TeV}$ with the ATLAS detector", Phys.Rev. D85 (2012) 092014, arXiv:1203.3161 [hep-ex] .

[18] CMS Collaboration, "Measurement of Rapidity Distributions for a Z Boson or a Photon in Association with a Single Jet in pp Collisions at sqrt(s)= 7 TeV”, Tech. Rep. CMS-PAS-SMP-12-004, CERN, Geneva, 2013.

[19] CMS Collaboration, "Measurement of the transverse momentum distributions of Z Bosons decaying to dimuons in pp collisions at sqrt(s)=8 TeV”, Tech. Rep. CMS-PAS-SMP-12-025, CERN, Geneva, 2013.

[20] ATLAS Collaboration, G. Aad et al., "Measurement of angular correlations in Drell-Yan lepton pairs to probe $\mathrm{Z} /$ gamma* boson transverse momentum at $\operatorname{sqrt}(\mathrm{s})=7 \mathrm{TeV}$ with the ATLAS detector", Phys.Lett. B720 (2013) 32-51, arXiv:1211.6899 [hep-ex] .

[21] C. Balazs, J.-w. Qiu, and C. Yuan, "Effects of QCD resummation on distributions of leptons from the decay of electroweak vector bosons”, Phys.Lett. B355 (1995) 548-554, arXiv : hep-ph/ 9505203 $[\mathrm{hep}-\mathrm{ph}]$.

[22] ATLAS Collaboration, G. Aad et al., "Study of jets produced in association with a $W$ boson in $p p$ collisions at $\sqrt{s}=7 \mathrm{TeV}$ with the ATLAS detector", Phys.Rev. D85 (2012) 092002, arXiv:1201.1276 [hep-ex].

[23] CMS Collaboration, S. Chatrchyan et al., "Jet Production Rates in Association with $W$ and $Z$ Bosons in $p p$ Collisions at $\sqrt{s}=7 \mathrm{TeV}$ ", JHEP 1201 (2012) 010, arXiv:1110.3226 [hep-ex] . 
[24] ATLAS Collaboration, G. Aad et al., "Measurement of the production cross section of jets in association with a $\mathrm{Z}$ boson in pp collisions at $\sqrt{s}=7 \mathrm{TeV}$ with the ATLAS detector", arXiv:1304.7098 [hep-ex].

[25] S. Frixione and B. R. Webber, "Matching NLO QCD computations and parton shower simulations", JHEP 0206 (2002) 029, arXiv: hep-ph/ 0204244 [hep-ph ] .

[26] M. L. Mangano, M. Moretti, F. Piccinini, R. Pittau, and A. D. Polosa, “ALPGEN, a generator for hard multiparton processes in hadronic collisions”, JHEP 0307 (2003) 001, arXiv: hep-ph / 0206293 $[$ hep-ph].

[27] H. Ita, Z. Bern, L. Dixon, F. Febres Cordero, D. Kosower, et al., "Precise Predictions for Z + 4 Jets at Hadron Colliders", Phys.Rev. D85 (2012) 031501, arXiv:1108.2229 [hep-ph] .

[28] ATLAS Collaboration, G. Aad et al., "Measurement of kT splitting scales in W->lv events at sqrt(s)=7 TeV with the ATLAS detector", Eur.Phys.J. C73 (2013) 2432, arXiv: 1302.1415 [hep-ex].

[29] CMS Collaboration, S. Chatrchyan et al., "Event shapes and azimuthal correlations in $Z+$ jets events in $p p$ collisions at $\sqrt{s}=7$ TeV”, Phys.Lett. B722 (2013) 238-261, arXiv:1301.1646 [hep-ex].

[30] S. Alioli, P. Nason, C. Oleari, and E. Re, "Vector boson plus one jet production in POWHEG", JHEP 1101 (2011) 095, arXiv:1009.5594 [hep-ph].

[31] ATLAS Collaboration, G. Aad et al., "Measurement of the cross-section for W boson production in association with b-jets in pp collisions at $\operatorname{sqrt}(\mathrm{s})=7 \mathrm{TeV}$ with the ATLAS detector", arXiv:1302.2929 [hep-ex].

[32] CMS Collaboration, "Measurement of the production cross section of W bosons in association with two b jets in pp collisions at sqrt(s)=7 TeV”, Tech. Rep. CMS-PAS-SMP-12-026, CERN, Geneva, 2013.

[33] ATLAS Collaboration, G. Aad et al., "Measurement of the cross-section for $b^{-}$jets produced in association with a $Z$ boson at $\sqrt{s}=7 \mathrm{TeV}$ with the ATLAS detector", Phys.Lett. B706 (2012) 295-313, arXiv:1109.1403 [hep-ex].

[34] CMS Collaboration, "Measurement of the Z/gamma* + bb-jets cross section in pp collisions at sqrt(s) $=7$ TeV”, Tech. Rep. CMS-PAS-SMP-13-004, CERN, Geneva, 2013.

[35] J. Alwall, M. Herquet, F. Maltoni, O. Mattelaer, and T. Stelzer, "MadGraph 5 : Going Beyond", JHEP 1106 (2011) 128, arXiv:1106.0522 [hep-ph]. 